\title{
Disturbance Attenuation in Classes of Uncertain Linear Hybrid Systems
}

\author{
Hai Lin and Panos J. Antsaklis
}

\begin{abstract}
In this paper, we study the disturbance attenuation properties for some classes of discrete-time uncertain piecewise linear hybrid/switched systems, which are affected by both time-variant parameter variations and persistent exterior disturbances. The problem of determining nonconservative bounds on the $l^{\infty}$ induced gain from the disturbance to controlled output for the closed-loop uncertain linear hybrid system is investigated. A procedure is given to determine such minimal $l^{1}$ norm of the uncertain piecewise linear systems. However, the termination of the procedure developed for general uncertain piecewise linear systems is not guaranteed. Therefore, it is important to specify a subclass of piecewise linear systems whose $l^{1}$ norm can be determined in finite number of steps. For such a purpose, we simplify the discrete event dynamics of the uncertain hybrid systems and obtain its subclass called uncertain switched linear systems. It is shown that the uncertain switched linear systems' $l^{1}$ norm can be determined in finite number of steps.
\end{abstract}

\section{INTRODUCTION}

The dynamic uncertainty and robust control of hy$\mathrm{brid} / \mathrm{switched} \mathrm{systems} \mathrm{is} \mathrm{a} \mathrm{highly} \mathrm{promising} \mathrm{and} \mathrm{challenging}$ field, which has been attracting more and more researchers' attention. However, the literature on this topic is relatively sparse. Some of the contributions include modelling uncertain hybrid/switched systems, reachability analysis, stability analysis and so on. For example, impulse differential inclusions were proposed as a modeling framework for uncertain hybrid/switched systems in [2], and some theoretic results for viability and invariance analysis in classical differential inclusions were extended to the impulse differential inclusions. Some reachability analysis results for uncertain hybrid/switched systems have appeared in [15], which was based on the backward reachability analysis techniques. There are also a few related works on robust controller design. In [17], the authors gave an abstract algorithm, based on modal logic formalism, to design the switching mechanism among a finite number of continuous variable systems. It was shown that the closed-loop system formed a hybrid automaton and satisfied certain specifications robustly. In [16], a robust tracking and regulation controller was designed for uncertain piecewise linear hybrid systems based on linear programming techniques. In [10], the authors proposed logically supervised switching multiple controllers to control uncertain dynamical systems, and the closed-loop system formed a class of uncertain switched systems. The advantages of switching controllers over classical adaptive

The partial support of the National Science Foundation (NSF ECS9912458 \& CCR01-13131) is gratefully acknowledged. The first author appreciates the support from Center of Applied Mathematics Fellowship (2003-04), University of Notre Dame.

H. Lin and Panos J. Antsaklis are both with the Department of Electrical Engineering, University of Notre Dame, Notre Dame, IN 46556, USA. Email: $\{$ hlin1, antsaklis. 1$\}$ and.edu controllers were discussed in [10] as well. These advantages partially explain the increasing interest in switched systems during the past decade. For robust stabilization of uncertain switched systems, a quadratic stabilizing switching law was designed for polytopic uncertain switched systems based on LMI techniques in [21].

In this paper, we will focus on the induced gain analysis for uncertain linear hybrid/switched systems. There are some related works in the literature on analyzing the induced gain in switched systems. In [19], the $\mathcal{L}_{2}$ gain of continuous-time switched linear systems was studied by an average dwell time approach incorporated with a piecewise quadratic Lyapunov function, and the results were extended to discrete-time case in [20]. In [9], the root-meansquare (RMS) gain of a continuous-time switched linear system with slow switchings was computed in terms of the solutions to a collection of Riccati equations. However, these robust performance problems considered are both in the signal's energy sense, and assume that the disturbances are constrained to have finite energy, i.e. bounded $\mathcal{L}_{2}$ norm. In practice, there are disturbances that do not satisfy this condition and act more or less continuously over time. Such disturbances are called persistent [7], and can not be treated in the above framework. Therefore, in this paper we consider $l^{\infty}$ induced gain to deal with the robust performance problems in the signal's magnitude sense, i.e. time domain specifications. Moreover, we explicitly consider dynamic uncertainty in the hybrid system model here. Dynamics uncertainty in the plant model is one of the main challenges in control theory, and being able to deal with dynamical uncertainties explicitly is of practical importance.

This paper is organized as follows. In Section II, we first define the uncertain piecewise linear systems, and then formulate the corresponding $l^{\infty}$ induced gain analysis problem. The goal is to determine the optimal disturbance attenuation level that can be achieved by the available control mechanism. In Section IV, the $l^{\infty}$ induced gain analysis problem is transformed into checking the robust controlled invariance of the disturbance attenuation performance level set. Invariant set theory has been studied in the literature for decades, see for example [6], [4], [3] and references therein. The invariance checking and calculation are based on backward reachability analysis and symbolic model checking method, which are studied in Section III. Based on the geometric condition for robust controlled invariance, a condition for checking whether there exist control mechanisms to achieve a given disturbance attenuation level is given. In Section IV, a bisection based procedure is proposed to determine a nonconservative bound on the optimal disturbance attenuation level that can be achieved for the uncertain piecewise 
linear systems. However, the termination of the proposed procedure in finite number of steps is not guaranteed. This is mainly because of the fact that the reachability problem is undecidable for general hybrid systems [1]. Hence, an important question is to specify the decidable class for the robust performance analysis problem. In Section V, we will focus on the decidability of the $l^{\infty}$ disturbance attenuation problem, and specify a decidable subclass of the uncertain piecewise linear systems, called uncertain switched linear systems. The determination of an nonconservative upper bounds on the $l^{\infty}$ induced gain for uncertain switched linear systems can terminate in finite number of steps. It should be pointed out that the $l^{\infty}$ disturbance attenuation problem for a class of switched linear systems without continuous inputs were previously studied in [13]. Section V is an extension of [13] to a more general case, namely to the case of continuous control law and switching signal co-design.

Notation: The letters $\mathcal{E}, \mathcal{P}, \mathcal{S} \cdots$ denote sets, $\partial \mathcal{P}$ the boundary of set $\mathcal{P}$, and $\operatorname{int}\{\mathcal{P}\}$ its interior. A polytope (bounded polyhedral set) $\mathcal{P}$ will be presented either by a set of linear inequalities $\mathcal{P}=\left\{x: F_{i} x \leq g_{i}, i=\right.$ $1, \cdots, s\}$, and compactly by $\mathcal{P}=\{x: F x \leq g\}$, or by the dual representation in terms of the convex hull of its vertex set $\left\{x_{j}\right\}$, denoted by $\operatorname{Conv}\left\{x_{j}\right\}$. For $x \in \mathbb{R}^{n}$, the $l^{1}$ and $l^{\infty}$ norms are defined as $\|x\|_{1}=\sum_{i=1}^{n}\left|x_{i}\right|$ and $\|x\|_{\infty}=\max _{i}\left|x_{i}\right|$ respectively. $l^{\infty}$ denotes the space of bounded vector sequences $h=\left\{h(k) \in \mathbb{R}^{n}\right\}$ equipped with the norm $\|h\|_{l_{\infty}}=\sup _{i}\left\|h_{i}(k)\right\|_{\infty}<\infty$, where $\left\|h_{i}(k)\right\|_{\infty}=\sup _{k \geq 0}\left|h_{i}(k)\right|$.

\section{Problem Formulation}

We consider discrete-time uncertain piecewise linear systems of the form

$$
\begin{array}{r}
x(t+1)=A_{q}(w(t)) x(t)+B_{q}(w(t)) u(t)+E d(t), \\
t \in \mathbb{Z}^{+}, \quad \text { if } x \in \mathcal{P}_{q}
\end{array}
$$

where $x(t) \in \mathbb{R}^{n}$ is the state variable, $u(t) \in \mathcal{U}_{q} \subset \mathbb{R}^{m}$ is the control input, and the disturbance input $d(t)$ is contained in $\mathcal{D} \subset \mathbb{R}^{r}$, the $l^{\infty}$ unit ball, i.e. $\mathcal{D}=\left\{d:\|d\|_{l^{\infty}} \leq 1\right\}$. The uncertain parameter $w(t) \in \mathcal{W} \subset \mathbb{R}^{v}$. It is assumed that $\mathcal{U}_{q}$ and $\mathcal{W}$ are polytopes assigned to each mode $q$.

Let the finite set $Q$ stand for the collection of discrete modes $q$. The partition of the state space $\mathcal{X}$ is given as a finite set of polyhedra $\left\{\mathcal{P}_{q}: q \in Q\right\}$, where $\mathcal{P}_{q} \subseteq \mathcal{X}$ and $\bigcup_{q \in Q} \mathcal{P}_{q}=\mathcal{X}$. The continuous variable dynamics of mode $q$ is defined by the parametric uncertain matrices $A_{q}(w)$, $B_{q}(w)$ and constant matrix $E$, and it is assumed that the entries of $A_{q}(w)$ and $B_{q}(w)$ are continuous functions of $w$ for every mode $q$.

A possible evolution of the uncertain piecewise linear systems from a given initial condition $x_{0} \in \mathcal{X}$ can be described as follows. First, there exists at least one discrete mode $q_{0} \in Q$ such that $x_{0} \in \mathcal{P}_{q_{0}}$, and the mode $q_{0}$ is called the feasible mode for state $x_{0}{ }^{1}$. Then the next continuous variable state is given by the transition $x_{1}=$ $A_{q_{0}}(w) x_{0}+B_{q_{0}}(w) u+E d$ for some possible $w \in \mathcal{W}$, $d \in \mathcal{D}$ and specific $u \in \mathcal{U}_{q_{0}}$. Then the above procedure is repeated for state $x_{1}$ to determine the next possible state $x_{2}$, and so on.

Associated with the uncertain piecewise linear system (1), a controlled output $z(t)$ is considered.

$$
z(t)=C(w(t)) x(t)
$$

where $C(w) \in \mathbb{R}^{p \times n}$ and $z(t) \in \mathbb{R}^{p}$. It is also assumed that the entries of $C(w)$ are continuous functions of $w$.

For this uncertain piecewise linear system (1)-(2), we are interested in determining a non-conservative bound for the $l^{\infty}$ induced norm from $d(t)$ to $z(t)$, which is defined as

$$
\begin{aligned}
\mu_{\text {inf }}=\inf \left\{\mu \mid \exists q(t) \in Q, u(t) \in \mathcal{U}_{q(t)} \quad:\right. & \|z(t)\|_{l \infty} \leq \mu, \\
& \left.\forall\|d(t)\|_{l^{\infty}} \leq 1\right\}
\end{aligned}
$$

The first problem considered in this paper can be formulated as follows.

Problem: Given the uncertain piecewise linear system (1)(2), determine the minimal $l^{\infty}$ induced gain from $d(t)$ to $z(t)$ that can be achieved by some admissible control law.

The basic idea employed in this paper is to translate the required level of performance into constraints on the controlled system, which can be dealt with by the invariant set theory. Therefore, we introduce the controlled robust invariant set for the uncertain hybrid systems as follows.

Definition 1: The set $\Omega \subset \mathcal{X}$ is controlled robust invariant for the uncertain piecewise linear system (1)-(2) if $\forall x_{0} \in \Omega$, there exists feasible modes and admissible control inputs, such that $x(t) \in \Omega, \forall t \geq 0$, despite disturbances and uncertainties.

In this paper, the invariance checking and calculation for $\Omega$ is based on the backward reachability analysis and robust predecessor operator, which will be introduced and developed for the uncertain piecewise linear system in the next section.

\section{Robust BACKWARD REACHABILITy ANALYSIS}

\section{A. Robust One-Step Predecessor Set}

The basic building block to be used for backward reachability analysis is the robust one-step predecessor operator, which is defined below.

Definition 2: The robust one-step predecessor set, $\operatorname{pre}(\Omega)$, is the set of states in $\mathcal{X}$, for which admissible control inputs exist and drive these states into $\Omega$ in one step, despite disturbances and uncertainties, i.e.

$$
\begin{aligned}
& \operatorname{pre}(\Omega)=\left\{x(t) \in \mathcal{X} \mid \exists q \in Q, u(t) \in \mathcal{U}_{q}: x(t) \in \mathcal{P}_{q},\right. \\
& \left.A_{q}(w) x(t)+B_{q}(w) u(t)+E d(t) \in \Omega, \forall d(t) \in \mathcal{D}, w \in \mathcal{W}\right\}
\end{aligned}
$$

\footnotetext{
${ }^{1}$ In the definition here, it is not required that the partition $\mathcal{P}_{q}$ have mutually empty intersections. Therefore, for the initial state $x_{0}$ there may exists more than one feasible discrete modes. For such case, it is assumed the current active mode, $q_{0}$ here, is selected from these feasible modes according to certain criteria or just randomly.
} 
We can also define the one-step predecessor set under the $q$-th mode, $\operatorname{pre}_{q}(\Omega)$, as the set of all states $x \in \mathcal{P}_{q}$, for which an admissible control input $u \in \mathcal{U}_{q}$ exists and guarantees that the system will be driven to $\Omega$ by the transformation $A_{q}(w) x+B_{q}(w) u+E d$ for all allowable disturbances and uncertainties.

Proposition 1: 2 The robust one-step predecessor set $\operatorname{pre}(\Omega)$ for an uncertain piecewise linear system can be computed as follows:

$$
\operatorname{pre}(\Omega)=\bigcup_{q \in Q} \operatorname{pre}_{q}(\Omega)
$$

Therefore, we only need to calculate the one-step predecessor set for each $q$-th subsystem.

\section{B. Predecessor Sets for Subsystems}

The difficulty of the calculation of $\operatorname{pre}_{q}(\Omega)$ mainly comes from the fact that the region $\Omega$ is usually non-convex. Even if one starts with convex sets, it usually deduces non-convex sets for piecewise linear systems after one-step predecessor operation. Because of the non-convexity, some of the linearity and convexity arguments do not hold and extra care should be taken.

In the sequel, we will focus on polytopic uncertainty in $A_{q}(w)$ and $B_{q}(w)$ for every mode $q \in Q$. It is assumed that

$$
A_{q}(w)=\sum_{k=1}^{v_{q}} w_{q}^{k} A_{q}^{k}, B_{q}(w)=\sum_{k=1}^{v_{q}} w_{q}^{k} B_{q}^{k},
$$

where $w_{q}^{k} \geq 0$ and $\sum_{k=1}^{v_{q}} w_{q}^{k}=1$. The pair $\left(A_{q}(w), B_{q}(w)\right)$ represents the model uncertainty which belongs to the polytopic set $\operatorname{Conv}\left\{\left(A_{q}^{k}, B_{q}^{k}\right), k=1, \cdots, v_{q}\right\}$ for each mode $q \in Q$. This is referred to as polytopic uncertainty and provides a classical description of model uncertainty. Similarly, we assume polytopic uncertainty in $C(w)$ as well, in particular

$$
C(w)=\sum_{l=1}^{N} w_{l} C^{l}
$$

where $w_{l} \geq 0$ and $\sum_{l=1}^{N} w_{l}=1$. Notice that the coefficients $w_{k}$ and $w_{l}$ are unknown and possibly time varying.

Under the polytopic uncertainty assumption, the calculation of the predecessor set for piecewise linear sets can be simplified, as implied by the following proposition.

Proposition 2: ${ }^{2}$ For polytopic uncertain piecewise linear systems, the robust one-step predecessor set for an assigned piecewise linear set $\Omega$ (may be non-convex) under the $q$-th subsystem can be calculated as

$$
\operatorname{pre}_{q}(\Omega)=\bigcap_{k=1}^{v_{q}} \operatorname{pre}_{q}^{k}(\Omega)
$$

where $\operatorname{pre}_{q}^{k}(\Omega)$ stands for the one-step predecessor operator of the $k$-th vertex state matrix $\left(A_{q}^{k}, B_{q}^{k}\right)$, i.e. $\operatorname{pre}_{q}^{k}(\Omega)=$

\footnotetext{
${ }^{2}$ Proof is omitted here for space limit, see [16] for details.
}

$\left\{x \in \mathcal{P}_{q} \mid \exists u \in \mathcal{U}_{q}: A_{q}^{k} x+B_{q}^{k} u+E d \in \Omega, \forall d \in \mathcal{D}_{q}\right\}$, for $1 \leq k \leq v_{q}$.

Therefore, we derived the relationship between robust one-step predecessor operator for the polytopic uncertain systems, $\operatorname{pre}_{q}(\cdot)$, and the one-step predecessor set of the vertex dynamics, $\operatorname{pre}_{q}^{k}(\cdot)$ for $k=1, \cdots, v_{q}$. It turns out that the robust one-step predecessor set for a piecewise linear set $\Omega$ under polytopic uncertain linear dynamics can be boiled down to the finite intersection of one-step predecessor sets corresponding to the dynamic matrix polytope vertices, which have no parametric uncertainty. The predecessor set under deterministic linear dynamics, $\operatorname{pr}_{q}^{k}(\Omega)$, has been studied extensively in the literature and can be computed by Fourier-Motzkin elimination [18] and linear programming techniques, see e.g. [4], [11].

Proposition 3: ${ }^{2}$ The robust one-step predecessor set for a (non-convex) piecewise linear set $\Omega$, pre $(\Omega)$, can be written as finite union of polyhedra.

Although the convexity is not preserved under the onestep predecessor operation, the piecewise linearity remains unchanged as Proposition 3 implies. Therefore, one can apply the predecessor operation recursively, which will be explored in the next section.

\section{Hybrid Robust PERFormance AnAlysis}

In this section, we will determine the minimal $l^{\infty}$ induced gain from $d(t)$ to $z(t)$ that can be achieved by some admissible control laws for the closed-loop piecewise linear systems. For such purpose, we first introduce the performance level $\mu$ set as

$$
\begin{aligned}
\Omega_{\mu} & =\left\{x:\|C(w) x\|_{\infty} \leq \mu\right\} \\
& =\left\{x:\left\|\sum_{l=1}^{N} w_{l} C^{l} x\right\|_{\infty} \leq \mu\right\} \\
& =\bigcap_{l=1}^{N}\left\{x:\left\|C^{l} x\right\|_{\infty} \leq \mu\right\} \\
& =\bigcap_{l=1}^{N}\left\{x:\left[\begin{array}{c}
C^{l} \\
-C^{l}
\end{array}\right] x \leq\left[\begin{array}{c}
\bar{\mu} \\
\bar{\mu}
\end{array}\right]\right\}
\end{aligned}
$$

where $\bar{\mu}$ stands for a column vector with $\mu$ as its elements. $\Omega_{\mu}$ is finite intersection of polytopes containing the origin in their interior. Therefore, $\Omega_{\mu}$ is a polytope containing the origin in its interior.

A value $\mu<+\infty$ is said to be admissible if $\mu>\mu_{\text {inf }}$. Clearly, a sufficient condition for $\mu$ to be admissible is that the hybrid performance level set $\Omega_{\mu}$ is controlled robust invariant. Therefore, the $l^{\infty}$ induced gain analysis problem is transformed into checking the controlled robust invariance of the disturbance attenuation performance level set. The following is an important, well-known geometric condition [6] for a set to be controlled invariant.

Theorem 1: The set $\Omega_{\mu}$ is a controlled robust invariant set if and only if $\Omega_{\mu} \subseteq \operatorname{pre}\left(\Omega_{\mu}\right)$.

In general, a given set $\Omega_{\mu}$ is not controlled robust invariant. However, $\Omega_{\mu}$ may contain controlled robust invariant 
subsets. In other words, the sufficient condition for $\mu$ to be admissible maybe too conservative. In order to get necessary and sufficient condition for the admissibility of $\mu$, we introduce the following definition.

Definition 3: The set $\mathcal{C}_{\infty}\left(\Omega_{\mu}\right)$ is the maximal controlled robust invariant set contained in $\Omega_{\mu}$ for the uncertain piecewise linear system (1)-(2) if $\mathcal{C}_{\infty}\left(\Omega_{\mu}\right)$ is controlled robust invariant and contains all the controlled robust invariant sets contained in $\Omega_{\mu}$.

The existence and uniqueness of the maximal controlled robust invariant set $\mathcal{C}_{\infty}\left(\Omega_{\mu}\right)$ follow immediately from the fact that the union of two controlled robust invariant sets is still controlled robust invariant. In order to calculate the maximal controlled robust invariant set in $\Omega_{\mu}$, we introduce the one-step controllable set of $\Omega_{\mu}$ as

$$
\mathcal{C}_{1}\left(\Omega_{\mu}\right)=\operatorname{pre}\left(\Omega_{\mu}\right) \cap \Omega_{\mu} .
$$

It follows from Proposition 3 that the one-step controllable set $\mathcal{C}_{1}\left(\Omega_{\mu}\right)$ is a piecewise linear set if $\Omega_{\mu}$ is given as a piecewise linear set. Therefore, the one-step controllable set operator can be used recessively to define $i$-step controllable set $\mathcal{C}_{i}\left(\Omega_{\mu}\right)$ as follows.

$\mathcal{C}_{i}\left(\Omega_{\mu}\right)=\mathcal{C}_{1}\left(\mathcal{C}_{i-1}\left(\Omega_{\mu}\right)\right)=\operatorname{pre}\left(\mathcal{C}_{i-1}\left(\Omega_{\mu}\right)\right) \cap \mathcal{C}_{i-1}\left(\Omega_{\mu}\right)$,

for $i \geq 2$. The sequence of finite-step controllable sets $\mathcal{C}_{i}\left(\Omega_{\mu}\right)$ has the following property.

Proposition 4: The sequence of finite step controllable sets $\mathcal{C}_{i}\left(\Omega_{\mu}\right)$ is decreasing in the sense of

$$
\mathcal{C}_{i}\left(\Omega_{\mu}\right) \subseteq \mathcal{C}_{i-1}\left(\Omega_{\mu}\right)
$$

for $i \geq 1$ and $\mathcal{C}_{0}\left(\Omega_{\mu}\right)=\Omega_{\mu}$. The maximal controlled invariant set in $\Omega_{\mu}$ for the uncertain piecewise linear system (1) is given by

$$
\mathcal{C}_{\infty}\left(\Omega_{\mu}\right)=\bigcap_{i=0}^{\infty} \mathcal{C}_{i}\left(\Omega_{\mu}\right) .
$$

The proof can be adopted from the proof of Theorem 3.1 in [4], which is not difficult, so it is omitted here.

Based on the notation of the maximal controlled robust invariant set $\mathcal{C}_{\infty}(\Omega)$, we state now the basic result of this section which will be used to give a solution to the disturbance attenuation property analysis problem.

Proposition 5: A value $\mu<+\infty$ is admissible, i.e. $\mu>$ $\mu_{\text {inf }}$, if and only if the maximal controlled robust invariant subset of $\Omega_{\mu}, \mathcal{C}_{\infty}\left(\Omega_{\mu}\right)$, is non-empty.

This result suggests the following constructive procedure for finding a robust performance bound.

Procedure 1. Checking whether $\mu>\mu_{\text {inf }}$

1) Initialization: Set $i=0$ and $\operatorname{set} \mathcal{C}_{0}=\Omega_{\mu}$.

2) Compute the set $\mathcal{C}_{i+1}\left(\Omega_{\mu}\right)=\operatorname{pre}\left(\mathcal{C}_{i}\left(\Omega_{\mu}\right)\right) \cap \mathcal{C}_{i}\left(\Omega_{\mu}\right)$.

3) If $0 \notin \mathcal{C}_{i+1}$ then stop, the procedure has failed. thus, the output does not robustly meet the performance level $\mu$.

4) If the $\mathcal{C}_{i}\left(\Omega_{\mu}\right)=\mathcal{C}_{i-1}\left(\Omega_{\mu}\right)$, then stop, and set $\mathcal{C}_{\infty}\left(\Omega_{\mu}\right)=\mathcal{C}_{i}\left(\Omega_{\mu}\right)$.

5) Set $i=i+1$ and go to step 1 .
This procedure can then be used together with a bisection method on $\mu$ to approximate arbitrarily close to the optimal value $\mu_{\text {inf }}$, which solves the disturbance attenuation property analysis problem. In fact, if the procedure stops at step 3, which comes from the fact that if $\mathcal{C}_{\infty}\left(\Omega_{\mu}\right) \neq \emptyset$ then $0 \in \mathcal{C}_{i+1}$. we conclude that $\mu<\mu_{\text {inf }}$ and we can increase the value of the output bound $\mu$. Else, if the procedure stops at step 4, we have determined an admissible bound for the output, say $\mu>\mu_{\text {inf }}$, that can be decreased. The above discussion can be formalized as a bisection algorithm as follows:

Algorithm 1: Algorithm for Calculating $\mu_{i n f}$

1) Initialization: Choose the initial interval $\left[\mu_{1}, \mu_{2}\right]$ such that $\mu_{1} \leq \mu_{\text {inf }}<\mu_{2}$. Choose $\epsilon>0$, the tolerance level. If no knowledge of lower bounds of $\mu_{\text {inf }}$ is available, $\mu_{1}$ may be chosen as $\mu_{1}=\epsilon$.

2) While $\left(\mu_{2}-\mu_{1}\right)>\epsilon$, set $\mu_{3}=\frac{\mu_{1}+\mu_{2}}{2}$, and check whether $\mu_{3}>\mu_{\text {inf }}$ by the above Procedure. If $\mu_{3}>$ $\mu_{\text {inf }}$, then set $\mu_{2}=\mu_{3}$, else set $\mu_{1}=\mu_{3}$.

3) Output $\mu_{\text {inf }}=\frac{\mu_{1}+\mu_{2}}{2}$.

However, the reachability problem for general hybrid systems is undecidable, see for example [1]. Therefore, the bisection method on $\mu$ to approximate the optimal value $\mu_{\text {inf }}$ can not be guaranteed to terminate in finite number of steps. Nevertheless, the possibility of an endless loop can be averted by putting an a priori limit on the number of iterations or by employing a grid based approximation as a termination condition. We will focus on the decidability issue in the next section.

\section{Decidable Uncertain Switched Linear SYSTEMS}

In the previous section, we studied the robust performance analysis problem of the polytopic uncertain piecewise linear systems. However, the termination of the proposed procedure in finite number of steps is not guaranteed. Hence, an important question is to specify the decidable class for the robust performance problems. Two kinds of simplification may be employed to make the procedures decidable. One way to obtain such decidable class is to simplify the continuous variable dynamics, see for example [1]. However, this approach may not be attractive to control applications, where simple continuous variable dynamics may not be adequate to capture the system's dynamics. Alternatively, one may restrict the discrete event dynamics of the uncertain linear hybrid systems. In this section, we will focus on the decidability of the $l^{\infty}$ disturbance attenuation problem, and specify a decidable subclass of the uncertain piecewise linear systems, called uncertain switched linear systems. The decidability comes from the simplicity of the discrete event dynamics. In particular, for the switched linear systems, we do not consider partition of the state space $\mathcal{P}_{q}$. In other words, the transitions between modes may happen at any point in the state space. Notice that previous work along this line appeared in [13], in which a class of switched autonomous linear systems was 
investigated. This section considers a more general model, and extends the results in [13].

\section{A. Uncertain Switched Linear Systems}

In this section, we consider a family of discrete-time uncertain linear systems described by the following difference equations.

$x(t+1)=A_{q}(w) x(t)+B_{q}(w) u(t)+E d(t), \quad t \in \mathbb{Z}^{+}$

where $x(t) \in \mathbb{R}^{n}$ is the state variable, $u(t) \in \mathcal{U}_{q} \subset \mathbb{R}^{m}$ is the continuous control input, and the disturbance input $d(t)$ is contained in $\mathcal{D} \subset \mathbb{R}^{r}$, the $l^{\infty}$ unit ball. The continuous variable dynamics of mode $q$ is defined by the parametric uncertain matrices $A_{q}(w), B_{q}(w)$ and constant matrix $E$ for every mode $q$. The finite set $Q=\left\{q_{1}, q_{2}, \cdots, q_{n}\right\}$ is called the set of modes.

Combine the family of discrete-time uncertain linear systems (3) with a class of piecewise constant functions of time $s: \mathbb{Z}^{+} \rightarrow Q$. Then we can define the following timevarying system as a discrete-time switched linear system

$x(t+1)=A_{s(t)}(w) x(t)+B_{s(t)}(w) u(t)+E d(t), \quad t \in \mathbb{Z}^{+}$

The signal $s(t)$ is called a switching signal. Let us denote the collection of all possible switching signals as $S_{a}$, which is usually called arbitrary switching signals in the literature [12], [8].

Associated with the switched linear system (4), a controlled output $z(t)$ is considered.

$$
z(t)=C(w) x(t)
$$

where $C(w) \in \mathbb{R}^{p \times n}$ and $z(t) \in \mathbb{R}^{p}$. It is also assumed that the entries of $A_{q}(w), B_{q}(w)$ and $C(w)$ are continuous function of $w \in \mathcal{W}$, where $\mathcal{W} \subset \mathbb{R}^{v}$ is an assigned compact set.

For this switched linear system (4)-(5), we are interested in determining a non-conservative bound for the $l^{\infty}$ induced norm from $d(t)$ to $z(t)$, which is defined as

$$
\begin{aligned}
& \mu_{\text {inf }}=\inf \left\{\mu \mid \exists s \in S_{a}, \exists u(t) \in \mathcal{U}:\|z(t)\|_{l^{\infty}} \leq \mu,\right. \\
& \left.\forall w(t) \in \mathcal{W}, \forall d(t),\|d(t)\|_{l \infty} \leq 1\right\}
\end{aligned}
$$

It is known that [13] the switched system (4)-(5) has finite $l^{\infty}$ induced gain if and only if its autonomous system is asymptotically stable. Therefore, we restrict our search only in the collection of asymptotically stabilizing control signals, $(s(t), u(t))$, which is denoted as $S_{s} \times \mathcal{U}_{s}$. It is assumed that there exist asymptotically stabilizing control signals, $(s(t), u(t))$, namely $S_{s} \times \mathcal{U}_{s}$ is nonempty. In the sequel, we will develop a procedure to determine $\mu_{i n f}$ for uncertain switched linear systems (4)-(5).

\section{B. $l^{1}$ Norm for Switched Systems}

We first introduce the definition of controlled invariant set for the switched system (4).

Definition 4: Considering the switched system (4), a set $\mathcal{P} \subset \mathbb{R}^{n}$ is said to be controlled invariant for this switched system if for every initial condition $x(0) \in \mathcal{P}$, for every admissible disturbance $d(t) \in \mathcal{D}$ and parameter variation $w(t) \in \mathcal{W}$, there exist admissible control signal $u(t) \in \mathcal{U}$ and switching law $s \in S_{a}$, such that $x(t) \in \mathcal{P}$ for $t \geq 0$.

We then formalize the definition of limit set, $\mathcal{L}^{(s, u)}$, under given admissible control signal $u(t) \in \mathcal{U}$ and switching law $s \in S_{a}$.

Definition 5: The limit set $\mathcal{L}^{(s, u)}$ for the switched system (4), under given admissible control signal $u(t) \in \mathcal{U}$ and switching sequence $s \in S_{a}$, is the set of states $x$ for which there exist admissible sequence $w(t)$ and $d(t)$ and a nondecreasing time sequence $t_{k}$ (with $\lim _{k \rightarrow+\infty} t_{k}=+\infty$ ) such that

$$
\lim _{k \rightarrow+\infty} \Phi\left(0, t_{k}, s(\cdot), u(\cdot), w(\cdot), d(\cdot)\right)=x
$$

where $\Phi\left(0, t_{k}, s(\cdot), u(\cdot), w(\cdot), d(\cdot)\right)$ denotes the value at the instant $t_{k}$ of the solution of (4) originating at $x_{0}=0$ and corresponding to $s, u, w$ and $d$.

For the asymptotically stabilizing control signals $(s, u) \in$ $S_{s} \times \mathcal{U}_{s}$, we know that the limit set $\mathcal{L}^{(s, u)}$ has the following property [5].

Lemma 1: For the asymptotically stabilizing control law $(s, u)$, the limit set $\mathcal{L}^{(s, u)}$ is nonempty and the state evolution of the switched system (4), for every initial condition $x(0)$ and admissible sequence $w(t) \in \mathcal{W}$ and $d(t) \in \mathcal{D}$, converges to $\mathcal{L}^{(s, u)}$. Moreover, $\mathcal{L}^{(s, u)}$ is bounded and if $x(t) \in \mathcal{L}^{(s, u)}$ then $x(t+1)=A_{s(t)}(w) x(t)+B_{s(t)}(w) u(t)+$ $E d(t) \in \mathcal{L}^{(s, u)}$ for all possible $d(t) \in \mathcal{D}$, and $w \in \mathcal{W}$.

Next, we define the limit set for the switched linear system (4) as

$$
\mathcal{L}=\inf \left\{\bigcap_{(s, u) \in S_{s} \times \mathcal{U}_{s}} \mathcal{L}^{(s, u)}\right\}
$$

where the intersection is with respect to any finite collection of the admissible control laws $(s, u)$ that asymptotically stabilize the switched system (4). It can be shown that $\mathcal{L}$ has the property as follows. The proof of the following propositions can be adopted from the proofs in [13], so they are omitted here for space limit.

Proposition 6: The set $\mathcal{L}$ is bounded and nonempty. For every initial condition $x(0)$, admissible $w(t) \in \mathcal{W}$ and $d(t) \in \mathcal{D}$, there exists an admissible control laws $(s, u)$ such that the state evolution of the switched system (4) converges to $\mathcal{L}$. In addition, $\mathcal{L}$ is controlled invariant for the switched system (4).

It should be pointed out that the introduction of the limit set $\mathcal{L}^{(s, u)}$ and $\mathcal{L}$ is for the purpose of proving the decidability of the procedures for $l^{1}$ analysis later, namely the termination in finite number of steps. There is no necessary to calculate these limit set $\mathcal{L}^{(s, u)}$ or $\mathcal{L}$ to implement the procedures for the determination of induced gains.

Similarly, define the performance level $\mu$ set as

$$
\Omega_{\mu}=\left\{x:\|C(w) x\|_{\infty} \leq \mu\right\}
$$


Recursively define the sets $\mathcal{C}_{k}, k=0,1, \cdots$ as

$$
\mathcal{C}_{0}=\Omega_{\mu}, \mathcal{C}_{k}=\mathcal{C}_{k-1} \bigcap \operatorname{pre}\left(\mathcal{C}_{k-1}\right)
$$

where the predecessor set for the switched system (4), $\operatorname{pre}(\mathcal{C})$, is the set of states $x$ from which, despite disturbances and dynamic uncertainties, there exist a subsystem (switching signal $s$ ) and continuous control signal $u \in \mathcal{U}$ driving the states to $\mathcal{C}$ in one step.

By construction, $\mathcal{C}_{\infty}$ has the property that there exists a switching signal $s(t)$ and continuous control signal $u(t)$ with respect to which $\mathcal{C}_{\infty}$ is positive controlled invariant for the switched system (4). Also it can be shown that $\mathcal{C}_{\infty}$ is the maximal controlled invariant subset contained in $\Omega_{\mu}$. Then, given $\mu>0$, there exists a switching signal $s(t)$ and continuous control signal $u(t)$ such that the response of the switched system satisfies $\|z(t)\|_{l^{\infty}} \leq \mu$ for all $w(t) \in \mathcal{W}$ and $\|d(t)\|_{l^{\infty}} \leq 1$ if and only if the maximal controlled invariant subset contained in $\Omega_{\mu}, \mathcal{C}_{\infty}$, is nonempty and $0 \in$ $\mathcal{C}_{\infty} \subseteq \Omega_{\mu}$

We now give a proposition which guarantees that $\mathcal{C}_{\infty}$ can be finitely determined.

Proposition 7: If $\mathcal{L} \subset \operatorname{int}\left\{\Omega_{\mu}\right\}$ for some $\mu>0$, then there exists $\mathbf{k}$ such that $\mathcal{C}_{\infty}=\mathcal{C}_{\mathbf{k}}$ and this $\mathbf{k}$ can be selected as the smallest integer such that $\mathcal{C}_{\mathbf{k}+\mathbf{1}}=\mathcal{C}_{\mathbf{k}}$.

The calculation of $\mu_{i n f}$ for uncertain switched systems (4)-(5) can now be solved by determining the maximal controlled invariant set $\mathcal{C}_{\infty}$ in $\Omega_{\mu}$ for several values of $\mu$ and checking whether or not it contains the origin. Note that in both cases we get an answer in a finite number of steps, although there is no a-priori bound for such a number. In the first case this is due to the above theorem. In the second case, this follows by the fact that the sequence of closed sets $\mathcal{C}_{\mathbf{k}}$ is ordered by inclusion and $\mathcal{C}_{\infty}$ is their intersection. Thus $0 \notin \mathcal{C}_{\infty}$ if and only if $0 \notin \mathcal{C}_{\mathbf{k}}$ for some $k$. These results suggest that the bisection algorithm (Algorithm 1) can be employed to approximate arbitrarily close to the optimal value $\mu_{\text {inf }}$.

\section{CONCLUDING REMARKS}

In this paper, we put the robust performance analysis problems of uncertain linear hybrid/switched systems into the framework of invariant set theory. The robust performance problem was transformed into robustly controlled invariance problems for a specific region decided by the performance level. Based on the geometric condition for robust controlled invariance, a bisection based procedure was proposed to determine the optimal disturbance attenuation level $\mu_{i n f}$. The decidability issue of the robust performance analysis problem was discussed, and switched linear systems were specified as a decidable subclass of the uncertain piecewise linear hybrid systems. The decidability comes from the simplicity of the discrete event dynamics. It is worth to point out that this specific simple subclass of hybrid systems still can model a large class of practical systems, such as multi-controller supervisory control systems, controller failures, fault diagnosis and control reconfiguration etc. For example, in [15], [14], a class of networked control systems with uncertain delay and package dropout effects was modeled as such switched system.

\section{REFERENCES}

[1] R. Alur, T. Henzinger, G. Lafferriere, and G. J. Pappas, "Discrete abstractions of hybrid systems," Proccedings of the IEEE, vol. 88, no. 7, pp. 971-984, 2000.

[2] J. P. Aubin, J. Lygeros, M. Quincampoix, S. Sastry, and N. Seube, "Impulse differential inclusions: a viability approach to hybrid systems," IEEE Transations on Automatic Control, vol. 47, no. 1, pp. 2-20, 2002.

[3] D. P. Bertsekas, "Infinite-time reachability of state-space regions by using feedback control," IEEE Transactions on Automatic Control, vol. 17 , no. 5 , pp. 604-613, 1972.

[4] F. Blanchini, "Ultimate boundedness control for uncertain discretetime systems via setinduced Lyapunov functions," IEEE Transactions on Automatic Control, vol. 39, no. 2, pp. 428-433, 1994.

[5] F. Blanchini, S. Miani, and M. Sznaier, "Robust performance with fixed and worst case signals for uncertain time-varying systems," Automatica, vol. 33, no. 12, pp. 2183-2189, 1997.

[6] F. Blanchini, "Set invariance in control," Automatica, vol. 35, no. 11, pp. 1747-1767, 1999.

[7] M. A. Dahleh and I. Diaz-Bobillo, Control of Uncertain Systems: A Linear Programming Approach, Prentice Hall, 1994.

[8] R. A. Decarlo, M. S. Branicky, S. Pettersson, and B. Lennartson, "Perspectives and results on the stability and stabilizability of hybrid systems," Proccedings of the IEEE, vol. 88, no. 7, pp. 1069-1082, 2000.

[9] J. P. Hespanha, "Uniform stability of switched linear systems: Extensions of LaSalle's invariance principle," to appear in the IEEE Transactions on Automatic Control, May 2004.

[10] J. P. Hespanha, D. Liberzon, and A. S. Morse, "Overcoming the limitations of adaptive control by means of logic-based switching," Syst. \& Contr. Lett., vol. 49, no. 1, pp. 49-65, 2004.

[11] E. C. Kerrigan, Robust Constraint Satisfaction: Invariant Sets and Predictive Control, $\mathrm{PhD}$ thesis, University of Cambridge, UK, November 2000.

[12] D. Liberzon and A. S. Morse, "Basic problems in stability and design of switched systems," IEEE Control Systems Magazine vol. 19, no. 15 , pp. $59-70,1999$.

[13] H. Lin and P. J. Antsaklis, "Disturbance attenuation properties for discrete-time uncertain linear switched systems," in Proceedings of the 42nd IEEE Conference on Decision and Control, pp. 5289-5294, 2003.

[14] H. Lin, G. Zhai, and P. J. Antsaklis, "Robust stability and disturbance attenuation analysis of a class of networked control systems," in Proceedings of the 42nd IEEE Conference on Decision and Control, pp. 1182-1187, 2003.

[15] H. Lin and P. J. Antsaklis, " Robust Regulation of Polytopic Uncertain Linear Hybrid Systems with Networked Control System Applications", Chapter in Stability and Control of Dynamical Systems with Applications, D. Liu and P. J. Antsaklis Eds., Chp. 4, pp. 83-108, Birkhäuser, 2003.

[16] H. Lin and P. J. Antsaklis, "Robust Tracking and Regulation Control of Uncertain Piecewise Linear Systems," ISIS Technical Report, ISIS-2003-005, 2003.

Available at http://www.nd.edu/ isis/tech.html.

[17] T. Moor and J. M. Davoren, "Robust controller synthesis for hybrid systems using modal logic," in Proceedings of the 4th Hybrid Systems: Computation and Control International Workshop, pp. 433446, 2001.

[18] T. Motzkin, The theory of linear inequalities, Rand Corp., Santa Monica, CA, 1952.

[19] G. Zhai, B. Hu, K. Yasuda, and A. N. Michel, "Disturbance attenuation properties of timecontrolled switched systems," Journal of the Franklin Institute, vol. 338, pp. 765-779, 2001.

[20] G. Zhai, B. Hu, K. Yasuda, and A. N. Michel, "Qualitative analysis of discrete-time switched systems," in Proceedings of 2002 American Control Conference, vol.3, pp. 1880-1885, 2002.

[21] G. Zhai, H. Lin, and P. J. Antsaklis, "Quadratic stabilizability of switched linear systems with polytopic uncertainties," International Journal of Control, vol. 76, no. 7, pp. 747-753, 2003. 